\title{
Identification of factors and outcomes of trust in mobile supply chains
}

Nassim Ghondaghsaz

Boysen-TU Dresden-Research Training Group, TU Dresden, Dresden, Germany and Institute of Media and Communication Science, TU Dresden, Dresden, Germany, and

\author{
Sven Engesser \\ Institute of Media and Communication Science, TU Dresden, Dresden, Germany
}

\section{Abstract}

Purpose - The main purpose of this paper is the identification of the conceptualization of trust as well as its factors and outcomes in interorganizational relations in mobile supply chains (MSCs) in which multiple stakeholders collaborate.

Design/methodology/approach - The authors first used a comprehensive literature review to extract related factors and outcomes of trust. Second, the authors conducted semi-structured interviews in chemical and pharmaceutical companies in Germany. These organizations stand out as leaders in the concept of MSCs and have developed collaborations with various stakeholders.

Findings - Based on the results, a conceptual model has been developed that elaborates on the nature of trust and its factors and outcomes for cultivating trustful stakeholder collaboration. The study identifies six factors or approaches for building trust and two outcomes resulting from mutual trust.

Practical implications - The conceptual model presented in this study can serve as a basis for developing trust in MSCs. Interorganizational collaborations in MSCs are more successful when saturated with trust. The collaboration systems must allow the innovative organizations to create value through the adaptation of advanced technologies without failure.

Originality/value - The study adds to the body of knowledge in building trust in multiple stakeholder collaboration, particularly in innovative organizations which are involved with disruptive technologies.

Keywords Interorganizational trust, Information sharing, Stakeholders, Mobile supply chain, Supply chain collaboration, Sustainable production

Paper type Research paper

\section{Introduction}

The existence of trust, or lack thereof, has always been a critical factor for the success of supply chains. Partners need to establish a trustful environment to form strategic alliances (Frydlinger et al., 2019). Past research has described trust as a multidimensional concept due to the paradoxical nature of relationship marketing: to be a strong rival in the global economy requires one to be a trusted cooperator (within various trade networks) (Akrout and Diallo, 2017; Fawcett et al., 2017). With the right mentality, the development of functional competition becomes a mutual goal for the parties rather than an adversarial challenge (Agarwal and Narayana, 2020;

(C) Nassim Ghondaghsaz and Sven Engesser. Published in European Journal of Management and Business Economics. Published by Emerald Publishing Limited. This article is published under the Creative Commons Attribution (CC BY 4.0) licence. Anyone may reproduce, distribute, translate and create derivative works of this article (for both commercial and non-commercial purposes), subject to full attribution to the original publication and authors. The full terms of this licence may be seen at http:// creativecommons.org/licences/by/4.0/legalcode

The authors would like to thank the Boysen-TU Dresden-Research Training Group for the financial support that has made this publication possible. The Research Training Group is co-financed by TU Dresden and the Friedrich and Elisabeth Boysen-Foundation.

This paper forms part of a special section "Innovation, Management, and Governance for Sustainable Growth", guest edited by Alba Yela Aránega.

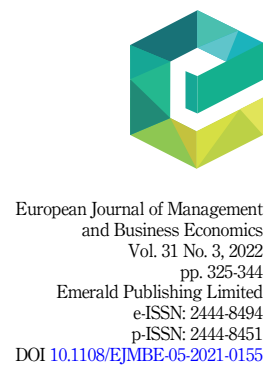

Received 27 May 2021 Revised 29 July 2021 16 August 2021

Accepted 21 August 2021 
EJMBE 31,3
Aslam et al., 2021). The absence of trust in interorganizational relationships, if not actively counteracted, can become an obstacle to collaboration in logistics, resulting in higher levels of transactional costs and lower levels of efficiency and productivity (Pomponi et al., 2015).

A current global megatrend is seeing manufacturers across the globe develop innovative production systems which are more environmentally friendly. Recent studies show that developments of concepts such as location independent manufacturing (Lohtander et al., 2017), modular production (Baldea et al., 2017) and factory-in-a-box (Jackson et al., 2008) represent just some of the new concepts within mobile supply chains (MSCs), where the production facilities can be transported in portable containers to provide real-time services at the location of the customer. This novel concept enables manufacturers to enhance adaptability, flexibility and reconfigurability of their production processes and decrease their transportation costs and CO2 footprint (Shahmoradi-Moghadam and Schönberger, 2021). Moreover, in MSCs, where advanced technologies are combined to "enable anything to be made anywhere" (Fox, 2019), a relatively high degree of risk and uncertainty can emerge due to the dynamic and complex features of this form of supply chain (Allman and Zhang, 2020).

Here, various involved stakeholders can pose significant challenges for building effective collaboration and trust, both of which are considered as critical for lowering the level of risk and uncertainty (Barrane et al., 2020). The difference between MSCs and the previously mentioned concepts is that manufacturing processes in MSCs are not in-house, which enables various stakeholders to take part in the decision-making process. This can lead to new business models in which the production lines can be shared among different stakeholders by means of leasing or renting production units. The complexity of a multi-stakeholder MSCs process is increased by the network of organizations, groups and individuals connected directly and indirectly through continuous or partial involvement in an MSCs (Ndubisi et al., 2020; Shahmoradi-Moghadam and Schönberger, 2021). Indeed, the MSCs concept depends strongly on the degree of relational stability and alliance performance in the network. The core of this relationship's durability is "trust-building" among various stakeholders (Barrane et al., 2020). Trust advances collaboration amongst partners and operational members and decreases ineffectiveness which arises from the opportunistic behavior of individual partners (Arvidsson and Melander, 2020; Fernando and Wulansari, 2020).

The recent literature on interorganizational trust has primarily focused on examining the importance of building trust and its impact on collaboration in supply chains. However, it fails to elaborate on the process of understanding, explaining or conceptualizing trust in building collaboration. Moreover, the existing literature lacks a complete understanding of how innovative companies who are applying Industry 4.0 in their production processes have managed to build and maintain trust with their partners (Savastano et al., 2018). In addition, while there is existing literature on how buyer-supplier trust is built in traditional supply chains (Sarkar et al., 2020), we do not know if the strategies for building trust and the consequences of maintaining trust would be different in the context of MSCs, where a digital environment is dominant. Nevertheless, a few studies have tried to explain how trust is understood and explained in this environment (Barrane et al., 2020; Sarkar et al., 2020), calling for more research to achieve a deeper understanding of the topic. To the best of our knowledge, there has been no exhaustive study on the discussion of trust dimensions and antecedents in the context of MSCs. Therefore, the present study fills the gap for the identification of the dimensions and antecedents of interorganizational trust in MSCs while building upon the available knowledge and distinguishing new opportunities for future research in this area. In this paper, we adopt a combined method. In the first stage of analysis, 17 empirical and review papers revealed various differences in both the operationalization and antecedents of building interorganizational trust. The results provided in this study clustered these dimensions and antecedents of trust to build a framework in order to understand the phenomenon of trust in the interorganizational context. In the second stage, a semi-structured interview with three 
qualified experts from the pharmaceutical industry in Germany allowed us to gain more knowledge about the nature of trust and its elements in the context of MSCs. The findings enable an evaluation of similarities and differences of the two approaches. This study will not, thus, yield statistically significant results. Rather, they will be valuable to clarify and/or extend the existing theory (Pomponi et al., 2015, p. 84). Therefore, the present study contributes to the study of supply chain trust in two ways. First, the main contribution of this study is the provision of a conceptual model that can serve as a basis for further empirical studies on interorganizational trust in the context of MSCs as well as multi-stakeholder collaboration. Second, we elaborate on the nature of trust, demonstrating dimensions and strategies for building and maintaining trust as well as the consequences of cultivating trust in both the existing literature and in MSCs. We also shed light on the differences between the results obtained from the existing literature and the interviews in terms of interorganizational trust. We conclude with specific recommendations and six strategies leading to the development of trust-enabled, collaborative interorganizational relationships.

The rest of this paper is as follows. The subsequent section discusses the review of the literature. This is followed by a section which describes the methodology behind the literature studies and semi-structured interviews. Finally, the paper ends with findings, a discussion and a conclusion.

\section{Theoretical background}

\subsection{Trust definition and antecedents}

Trust is a complex concept. It is a multidimensional and multifaceted communication phenomenon (Nematollahi, 2019; Paluri and Mishal, 2020). Various definitions of trust have been provided from different disciplines, such as marketing, management, supply chains, psychology, sociology and economics (see Table 1). From a marketing perspective, the seminal work by Morgan and Hunt (1994) examined the critical role of trust and commitment and their centrality in the success of relationship marketing. They argued that "trust exists when one party has confidence in an exchange partner's reliability and integrity". In the management discipline, Lui and Ngo (2004) suggested that trust is the expectation of a partner to fulfill a collaborative role in a risky situation, and (the reliability) of both the partner's intention to perform and its ability to do so. From a supply chain perspective, Lee and Zhong (2020) stated that trust between two stakeholders in a supply chain relationship contains two components, namely credibility and benevolence. Credibility indicates the firm's belief in the ability of the partner to perform their promise accurately, and benevolence refers to the belief that the supplier takes into consideration the buyer's welfare and interest in their interorganizational trust.

Wu et al. (2017) conceptualized trust as a two-dimensional structure, including calculative trust and relational trust. Akrout and Diallo (2017) categorized trust according to the different stages of trust development: calculative trust, cognitive trust and affective trust. Levels of trust can be classified as weak, semi strong and strong. Therefore, trust is defined as a willingness to expose yourself to risk (Ryciuk, 2017). In sociological research, trust entails the effect of history, culture and organizations on humans in a particular social context. Economists usually evaluate trust from a transaction cost and game theory perspective (Pech and Swicegood, 2013; Wu et al., 2017). Frydlinger et al. (2019) outlined the process of building long-term strategic partnerships under the foundation of trust through "formal relational contracts", in which five steps were drafted. These steps are related to establishing a problemsolving mentality rather than negotiations mentality, creating a jointly shared vision, adopting six "guiding principles: reciprocity, autonomy, honesty, loyalty, equity and integrity", lining up objectives and expectations and, finally, maintaining the relational contract through continuous monitoring.
Factors and outcomes of trust in MSCs 


\section{EJMBE 31,3}

328

\begin{tabular}{l} 
References \\
\hline Agarwal and \\
Narayana (2020 \\
Akrout and \\
Diallo (2017)
\end{tabular}

Chen et al. (2011)

Daudi et al.

(2016)

Ford et al. (2020)

Handfield and

Bechtel (2002)

Kwon and Suh (2004)

Lee and Zhong (2020)

Lui and Ngo

(2004)

Morgan and Hunt (1994)

Nicolaou and McKnight (2006)

Nyaga et al. (2010)
Goodwill/affect-based dimensions antagonistic/cooperative, distrust/trust and harmony/discord

Trust exists when a firm believes its partner is being honest and benevolent

Benevolence trust and competence trust

Goodwill, competency and contractual safeguards

Trust exists when one party has confidence in an exchange partner's reliability and integrity

One believes that the partner is honest, benevolent and competent

Keeping the other partner's best interest in mind and considering their welfare
Table 1.

Definition of trust, antecedents, and outcomes
Factors and outcomes

Factors: Information quality, information sharing, information frequency and relationship commitment Outcomes: Relationship satisfaction Factors: Reputation, conflict resolution, sympathy and shared values Outcomes: Relationship investment and confidential communication

Factors: Information availability, information sharing, information quality, country and behavioral uncertainty Outcome: Commitment

Factors: Information sharing, incentive alignment, decision synchronization and opportunism

Outcomes:-

Factors: Reputation, communication, service quality/satisfaction, shared norms and values, negative past behaviors, fairness and cooperation

Factors: Contracts, site specific asset, human specific asset and buyer dependent Outcomes: Responsiveness

Factors: Asset specificity, behavioral uncertainty, Information sharing, perceived satisfaction, partner's reputation and perceived conflict

Outcomes: Commitment

Factors: Bayer's dependence on supplier Outcomes: Contractual bonds and relational exchanges

Factors: Prior relationship, size difference and asset specificity

Outcomes: Completion time and performance satisfaction Factors: Opportunistic behavior, communication and shared values Outcomes: Relationship commitment Factors: Perceived information quality and disposition to trust

Outcomes: Intention to use and perceived risk

Factors: Information sharing and joint relationship effort

Outcomes: Satisfaction with relationship and performance 


\begin{tabular}{ll}
\hline References & Definitions of trust \\
\hline Ryciuk (2017) & $\begin{array}{l}\text { Trust is honesty, reliability, integrity, } \\
\text { dependability, promise keeping and } \\
\text { behavior consistent with expectations }\end{array}$
\end{tabular}

Salam (2017)

Wu et al. (2017)

Barrane et al. (2020)

Zhong et al. (2017)
Trust in the degree to which partners perceive each other as credible and benevolent

Calculative trust; relational trust

The study conceives trust to include two dimensions namely, credibility and benevolence

The extent of trust placed in the partner organization by the members of a focal organization
Factors and outcomes

Factors: Partner characteristics (reputation in quality and financial situations as well as well-known brand), relationship formalization (contracts) and poor bargaining position

Outcomes: Cooperation, goodwill manifestation and specific investments Factors: Mutuality and reciprocal loyalty Outcomes: Collaboration

Outcomes: Lower (relationship, task and process) conflict and added value

Factors: Long-term relationship, transparency, appropriate partner and informal conversations

Outcome: Trustful multi-stakeholder collaboration in digital environment Factors: Asset specificity, relationship duration, dependence and external uncertainty
Factors and outcomes of trust in MSCs

329

Table 1.

Researchers across different disciplines suggest a variety of factors and outcomes to understand trust development and maintenance (Ford et al., 2020). Chen et al. (2011) showed a positive effect of information availability, information sharing, information quality and behavioral uncertainty on trust. They indicated that the level of commitment is strongly related to the level of trust. Agarwal and Narayana (2020) examined the effect of three dimensions of relational communication (information sharing, information quality and information frequency) on social exchange constructs, namely trust, commitment and satisfaction. They concluded that these three dimensions are positively related to a buyer's satisfaction with the mediating role of trust.

\subsection{Mobile supply chain}

In production technology, the main focus is placed on technical aspects. Such methods are quite standardized. In industrial engineering and management, the primary focus in research is economy of scale in production. Environmental studies concentrate mainly on CO2 footprints and pollution emitted from industrial and production sources (Peltokoski et al., 2017). The MSCs concept tries to combine these research disciplines into a practical model.

The MSCs concept is an innovative concept that is not yet widely implemented. The main idea of MSCs is to build a production unit composed of various modules which can be mobile and movable, e.g. sea containers. There are several possible forms of the MSCs concept: it can be a movable assembly unit, mobile maintenance unit, a mobile service unit, a mobile power unit or a mobile production line. The concept of a mobile factory in itself is not a novel idea as it has been widely applied in mobile healthcare systems (e.g. mobile blood donations (Haghjoo et al., 2020)), energy and power (e.g. Siemens floating power plant), mobile libraries, fruit processing factories (Peltokoski et al., 2017) and mobile bio-oil refineries (Mirkouei et al., 2016).

In research, several background concepts have provided the foundation for MSC: industrial assembly and manufacturing systems (Rosell, 2004), distribution manufacturing systems (DMS) (Matt et al., 2015), mobile production and manufacturing systems (MMS) (Alix et al., 2019), factory-in-a-box (Hedelind et al., 2007) and location independent manufacturing 
EJMBE 31,3

(LIM) (Peltokoski et al., 2017). Several studies, when referring to mobile supply chain management $(\mathrm{mSCM})$ seemed occupied with the implementation of mobile and/or wireless technology in the supply chain (Cagliano et al., 2017; Chan and Yee-Loong Chong, 2013; Eng, 2006; Pan et al., 2013). However, in this study, mobility is related to production sites and facilities. It can be assumed that the use of wireless technologies is a necessary prerequisite for exchanging production information within MSCs.

According to (F3 factory, 2013) project research, the fundamentals and basis for implementing MSCs are mainly modular population and fully automated production lines Modularity has been defined as "the synergistic combination of fundamental functions (tasks) into a single equipment, e.g. reactive distillation, spinning disk reactors and membrane distillation" (Baldea et al., 2017). The concept of modular production has come about as one of the consequences of the modern process industry, whereby advanced technologies enable more flexible and customized responses to rapidly changing consumer demand. This results in greater diversity of product range, thereby increasing the demand for manufacturing technologies and equipment (Öberg, 2019). One significant benefit of modular production is the increased flexibility of production capacity, which can be influenced by fluctuating parallel modules. Moreover, modules can be easily orchestrated and combined, as well as easily installed due to practical plug-and-produce models.

Fox, (2015) has stated that three various types of mobile factories exist:

(1) "Individual mobile factory": located on a truck, used for shorter durations in a temporary location, e.g. fruit processing factories, mobile food truck and mobile libraries.

(2) "Sets of movable factories": including more than one production unit and used for longer durations in a location.

(3) "Modular factories": consisting of various modules in combination to provide one production line.

Selection of the appropriate type can be made based on the time, demand, products and production processes. The MSCs concept has many features which are similar to earlier concepts found in the literature, such as factory-in-a-box and LIM. For example, the similarities between the MSCs concept and previously developed concepts include the following: composed of standardized manufacturing modules, located in a movable container or truck and providing services to geographically dispersed end-consumers (See Figure 1), with both concepts requiring flexible and reconfigurable systems. MSCs democratizes previous concepts by transferring the decision-making to multiple stakeholders, meaning the decisions affecting mobile containers cannot be made by only one stakeholder.

\subsection{Importance of trust in mobile supply chains}

In the concept of MSCs, advanced technologies and Industry 4.0 foster an environment to easily collaborate and share information (Oghazi et al., 2018). The collaboration in MSCs is associated with a mobile production process, location, production volume (Becker et al., 2019), raw materials, market data, information exchange, knowledge sharing, employee rights, legislations (Peltokoski et al., 2017) and resources between the network stakeholders to attain economical co-creation value. The allocation of tasks and responsibilities among stakeholders ("corporate governance") has recently drawn attention as an issue of concern in many countries (Palaniappan, 2017). In this regard, previous studies have highlighted that multiple stakeholder collaborations have a positive impact on a firm's performance (Driessen and Hillebrand, 2013). 


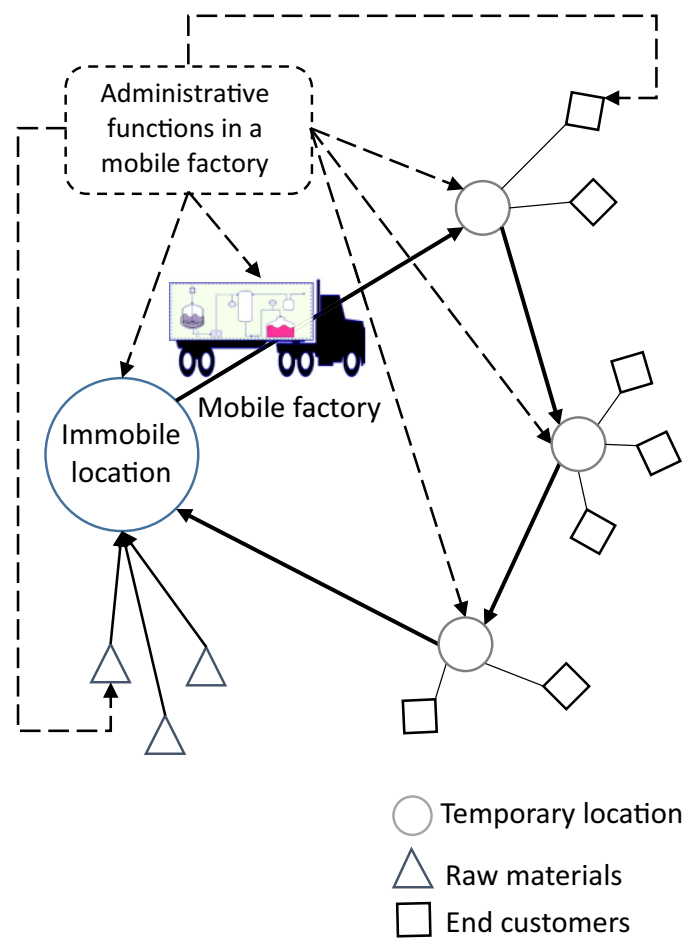

Factors and outcomes of trust in MSCs

331

Figure 1. Mobile Supply Chain's decentralized location and administrative functions

However, the collaboration system can be quite complicated and sometimes distorted by distrust and destructive conflicts (Barrane et al., 2020). The use of advanced technologies in MSCs represents an excellent opportunity to cope with this complexity and to establish a smart and novel manufacturing framework (Baldea et al., 2017; Oghazi et al., 2018). The design, material, components and manufacturing can each originate from different stakeholders (including from different countries), which makes controlling the process more complex (Lohtander et al., 2017). Indeed, the MSC's process depends strongly on the degree of relational stability and alliance performance within the network. The core of this relationship durability is "trustbuilding” among various stakeholders (Barrane et al., 2020; Yang et al., 2008). Moreover, a lack of trust is sensed more in complicated scenarios, where mobile factories change locations based on the decision of different stakeholders, and the process is carried out remotely without face-to-face communication between individuals and groups (Barrane et al., 2020; Wooliscroft and Ganglmair-Wooliscroft, 2018). Trust boosts cooperation between stakeholders and operational members, increases their commitments (Kwon and Suh, 2004) and decreases ineffectiveness arising from the opportunistic behavior of partners. Furthermore, previous studies have shown that trust becomes more crucial in relationships with greater risk and uncertainties, such as in the context of MSCs when the risks and transaction costs are very high (Ndubisi, 2010; Zafari et al., 2020). In sum, it has been noticed that companies which are considered trustworthy maintain higher levels of performance and can be associated with a long-term competitive advantage (Cappiello et al., 2020). The recent literature lacks a complete understanding of how innovative companies which have adopted Industry 4.0 and disruptive technologies 
EJMBE 31,3

332 within their production process are handling this situation. This study elaborates on the trust-building process among collaborative partners in such circumstances.

\section{Methodology}

In this study, we seek to determine major strategies for building trustful relationships in the concept of MSCs. For this purpose, we began with a comprehensive literature review to extract related factors and outcomes of trust. Second, the authors conducted a semistructured interview with key personnel in German chemical and pharmaceutical organizations in charge of MSCs. Semi-structured interviews contribute to the thorough exploration of the subject by posing questions that motivate the participant to provide their thoughts, experience and attitude (Barrane et al., 2020). Moreover, it helps researchers to collect data from a real-world perspective.

\subsection{Literature search}

We conducted a comprehensive search for empirical and review studies investigating a correlation between antecedents and consequences of trust, as well as providing a definition of the nature of trust. To identify related studies, we first searched computerized databases including Science Direct, ProQuest, Google Scholar and Emerald utilizing several keywords. These databases hold valuable publications in the area of supply chains, collaboration and trust. We included only published peer-reviewed journal articles. This was aimed at increasing the quality of the search following Delbufalo (2012). Appendix 2 shows the procedures utilized to identify the reviewed articles. The search was carried out in the mentioned databases using the search keywords, resulting in a total of 1,511 articles published between 1990 and 2021, inclusively. After eliminating the duplicated articles, we scanned the titles, abstracts and keywords of 1,454 articles based on their focus on trust in the context of supply chain management (SCM) or trust in Industry 4.0 supply chains. This step is preferred because the full-text contents of articles are less dedicated to defined keywords in comparison with those in title, abstract and keywords. This screening filters the previous 1,454 collected articles to 201 articles to be reviewed in full text. Subsequently, inclusion and exclusion criteria were utilized to screen the articles for relevancy. With the initial practical screening, each study had to cover interorganizational trust as a core of its analysis. Studies that considered interpersonal trust, intraorganizational or supplier-customer trust were excluded. Following this screening process, remaining studies had to investigate or evaluate either antecedents or outcomes of trust. Studies that solely covered the nature of trust were excluded. The screening process was followed by our methodological approach in which articles concentrating on optimization methods, game theory and blockchain were excluded. The reasoning here is that these articles do not focus on the nature of trust. We also applied a snowball approach and examined the reference lists of selected studies to ensure that no relevant papers were overlooked (Ford et al., 2020). This step added five more articles to our final number of reviewed articles. In total, we identified 17 studies for the analysis of antecedents, outcomes and trust definition.

\subsection{Qualitative interview}

Semi-structured interviews were also carried out to compare and verify the outcome of the literature review with multiple case studies, as well as to collect various opinions regarding the state of the art of MSCs. We selected two companies which are regarded as pioneers and leaders in innovative pharmaceutical production in Germany. The target population comprises of three top-ranked executives who hold top positions in their firms and are responsible for controlling the MSC and modular production. Since an important aspect of 
this research is to strengthen the German process industry, all experts have their main research area and headquarters in Germany. We guarantee the anonymity of all respondents throughout the study process by allocating non-descript alphabetical identifier codes to each participant. An interview guide with six open-ended questions was prepared to manage the discussions with the experts. The interview questions are provided in Appendix 1.

The objective of the interviews was to empirically examine the trust definition, as well as its factors and outcomes. The interview protocol was designed based on the literature and was used for all respondents. The semi-structured interviews began with a presentation of the concept of MSCs to familiarize the interviewee with the objectives and questions in the interview.

Interviews were held online due to COVID-19 restrictions and durations varied between $30 \mathrm{~min}$ and $1 \mathrm{~h}$. To ensure the accuracy of the data and to ease the coding and interpretation, all interviews were recorded and subsequently transcribed.

\subsection{Data analysis and interpretation}

The selected articles were analyzed to identify the nature and antecedents of trust in the existing literature. The analysis was carried out using the MaxQDA tool following procedures outlined by Kuckartz and Rädiker (2019). The coding process for the analysis was accomplished by allocating categories and concepts to the different sections related to the research purposes. The analysis was interpreted and summarized through a qualitative data set synthesis process. According to Olugboyega and Windapo (2019), data set synthesis makes it easier to understand a phenomenon through research collection. The synthesis process categorizes concepts into main subjects (nature of trust and antecedents) and identifies general opinions on the main subjects to ensure credibility. Following the literature review, we created a codebook and synthesized the responses. This enabled us to compare interviewees' responses and create categories to interpret the data. To increase the research reliability and validity, we controlled the interviews and documents repeatedly, compared them with the literature and asked an independent researcher, an expert in the field of modular production, to check them. Moreover, we created a database of collected data and findings (interview guide, interview description, online videos, transcription and codebooks). In the findings section, some relevant responses from the participants are included to enhance research validity.

\section{Findings and discussion}

\subsection{Results from the literature review}

Although the importance of trust has long been recognized in the literature related to interorganizational relationships, and various definitions of trust have been provided, three major dimensions of trust in the literature were mentioned quite often. The analysis was summarized graphically using a descriptive figure (see Figure 2). The three categories in terms of the nature of trust include honesty (35\%), benevolence (45\%) and competency (20\%). Percentages show how often a dimension of trust was mentioned in the studied articles. The scholars provided various definitions for trust, but, as we elaborated on the nature and conceptualization of trust, it seems that the contexts behind these definitions are similar, and therefore, they can be grouped together with other categories. Therefore, in the group of honesty, we can consider other operationalizations of trust, such as "sincere" and "stand by their word".

The group of benevolence can also be defined as "goodwill", "interested in the firm's welfare" and "no unexpected actions". Competency can also be understood as the ability to do what a partner needs to be done or fulfilling promised role obligations. Besides these three
Factors and outcomes of trust in MSCs 


\section{EJMBE 31,3}

\section{4}

Figure 2.

Conceptualizations of trust based on results from literature review

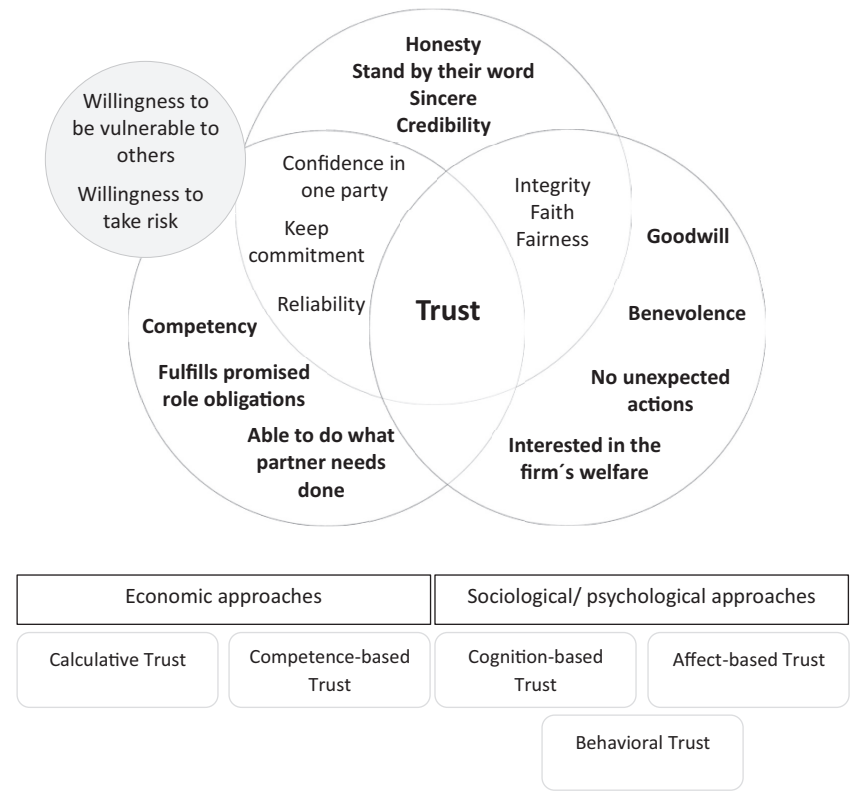

major groups, some scholars have identified trust as a willingness to be vulnerable to others. Moreover, various level-based definitions of trust have been considered, such as calculative trust, competence-based trust, affect-based trust and behavioral trust. The analysis suggests that calculative trust and competence-based trust are more concerned with economic approaches, while the others are more related to sociological/psychological approaches.

Moreover, the results of the literature review allowed for the proposal of various factors and outcomes related to establishing, evaluating, maintaining and developing trust in supply chain management. It seems that the multifaceted nature of trust can be observed in this stage as several constructs can have a reciprocal relationship with trust. For example, the relationship between trust and cooperation, trust and information sharing, and trust and conflict have all been suggested as being reciprocal. It shows that trust can be both a cause in some circumstances and partially an effect. This could explain why determining the nature of trust is a complicated concept to understand and study. In Figure 3, various antecedents and outcomes of trust are shown of which some belong to both groups. To summarize and analyze these antecedents and outcomes, we clustered four categories for factors (information, transaction cost, social exchange and governance) and four sections for outcomes (collaboration, cooperation, investment and responsiveness). Each factor and outcome has various components.

The following section presents the results of the expert interviews. These results enable a comparison with the existing literature and an evaluation of similarities and differences. This approach contributes to an elaboration of gaps in the literature where more research would be useful.

\subsection{Results from semi-structured interviews}

All participants admitted to the importance of including various partners in an MSC, but they also emphasized the challenges this entails. One of the respondents noted "our projects are very complex and we need good partners," while another said: “. . . it is really tough”. The aim 


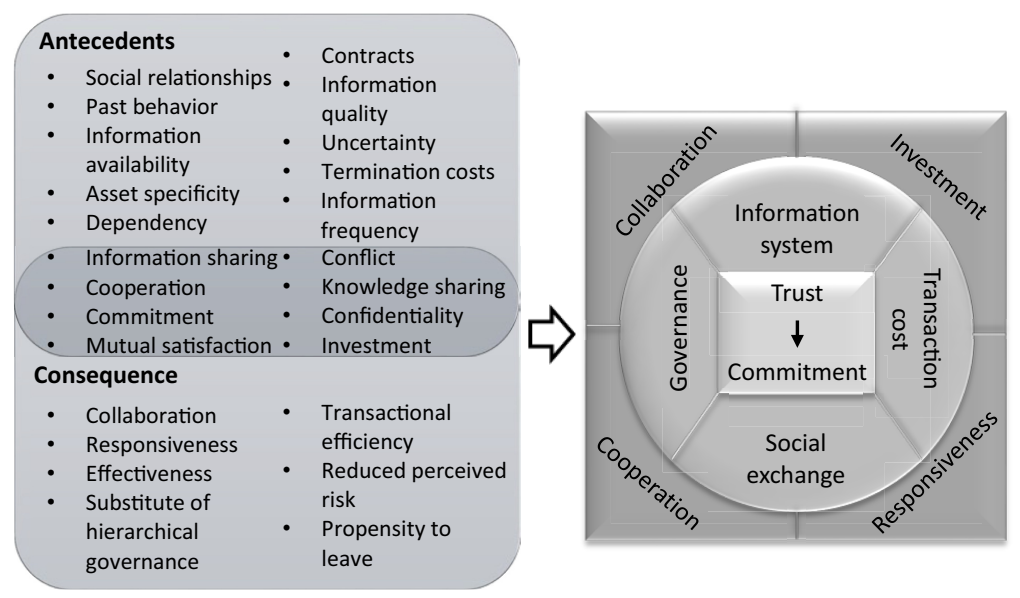

Factors and
outcomes of
trust in MSCs

335

of the semi-structured interviews conducted with representatives of pharmaceutical companies was the identification of the nature of trust and the antecedents that are significant for establishing and maintaining interorganizational trust in MSCs (one benefit of expert interviews is the uncovering of aspects not previously considered by the researchers). Figure 4 summarizes our results in terms of dimensions, antecedents and outcomes of interorganizational trust in the context of MSCs.

The empirical results of the study show that trust is determined by honesty, credibility and trustworthiness components in these organizations. One of the respondents shared a similar view: trust is "the feeling if the partner will live up to their promises". The results show that trust is mostly identified with performance according to promises, as well as confidence in one party. Another respondent remarked: "trust is defined as your partner delivering with agreed quality ... they deliver in the agreed time". The results confirmed the findings of previous studies (Nicolaou and McKnight, 2006; Ryciuk, 2017), which defined trust as

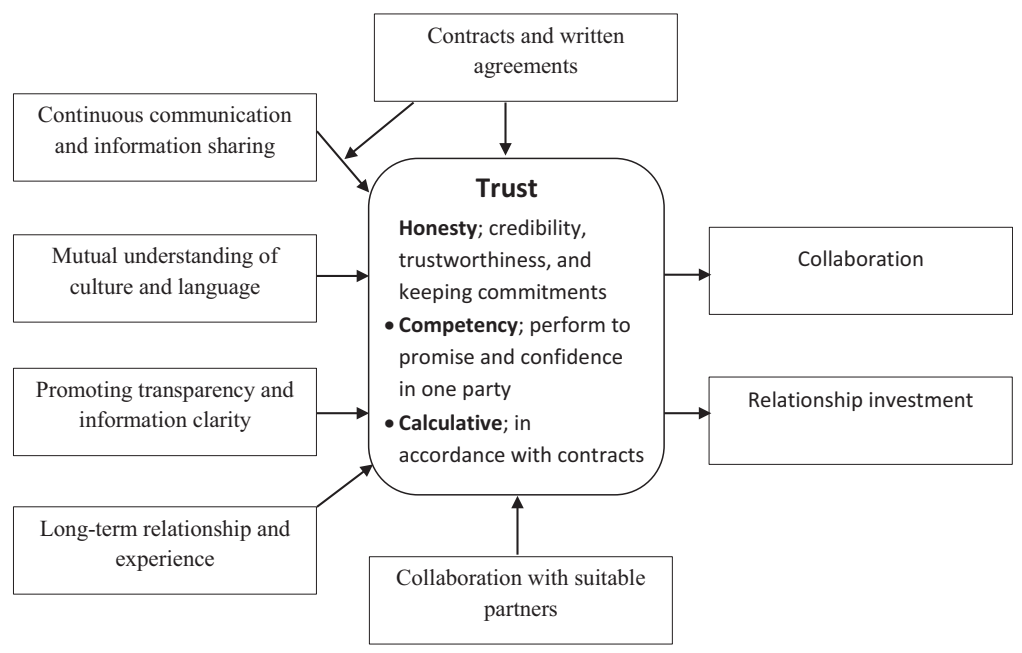

Figure 4.

Dimensions, factors and outcomes of interorganizational trust in mobile supply chains consequences of trust based on results from literature review 
EJMBE 31,3 honesty and integrity. Another respondent noted: "we have to get from the vendor what we want", which implies that trust can be defined as competency, keeping commitments, the ability to do what the partner needs to be done and fulfilling promised responsibilities properly. These results are consistent with some findings from the existing literature (Lee and Zhong, 2020). Trust also implies a belief that the partner will carry out their responsibilities according to the agreements and contracts. One of our respondents remarked: "If it is clearly defined in the contract that certain information is not shared, then it has no negative impact on trust". This illustrates the fact that an enormous portion of trust comes from contractual safeguards provided by these agreements. This component of trust has also been emphasized in previous studies (Akrout and Diallo, 2017; Beuve and Saussier, 2012). Calculative trust provides security in the presence of opportunistic behavior, thereby resulting in decreasing transaction costs in the relationships (Akrout and Diallo, 2017) and contributing to the maintenance of trust. Comparing the results from the semi-structured interviews with previous studies, it is obvious that the significant difference between traditional supply chains and MSCs in terms of trust dimensions is the benevolence component of trust, which was mainly emphasized in the existing literature but not mentioned by our respondents. Benevolence means the partner considers the goodwill and interest of the firm and acts accordingly. One major difference between the existing literature and the findings of our interviews is that benevolence is not as important as the other two trust components (honesty and competency). This finding mainly contradicts previous studies as benevolence was the most often mentioned component of trust. The reason for the lower importance of benevolence in the MSCs context could be the complex nature of these projects, which requires one party to stick to the contracts, have the ability to fulfill the firm's expectation and keep their promises, as one small act of opportunistic behavior can result in large risk exposure. To understand how partners can build and maintain trust in MSCs, the interviews followed up by asking the respondents about their approaches.

According to the interview participants, the main factors or approaches cultivating trust in interorganizational relationships within MSCs include:

(1) Continuous communication and information sharing: our respondents emphasized the importance of frequent sharing of information among partners in an MSC. However, the amount of shared information depends on the level of integration. In some scenarios, where a partner (supplier) produces a specific raw material for another partner through mobile manufacturing, they need a high level of interaction in which a great amount of information should be exchanged to provide effective communication in order to build trust. According to the type of information, one of our respondents remarked: “... the interaction could be intense because your production depends on information from my production stages. So what capacity I am running, how pure my product is, do I have any side components you know that basically makes my product dirty ... all these types of information would be required for you to run your asset in an optimal way". In another scenario, where the partner requires a product or a service that can be provided with the least amount of shared information, a lower level of integration emerges. As one of our respondents stated "... it is not uncommon that if you need pressurized air, so somebody is running a compressor for you, so that you get air as an energy supplier... X company would not let you know what exactly is happening in their mobile plant". In the literature (Chen et al., 2011; Daudi et al., 2016), the positive impact of information sharing on trust has been emphasized repeatedly, but these studies did not elaborate on scenarios where information is not shared. Subsequently, the reason for not sharing information and the effect that would have on trust have not been examined. Our results show that information sharing is essential for building trust in MSCs, but it depends on the level of 
integration experienced. In scenarios where information needs to be shared extensively, and a partner refrains from sharing, the level of trust will decrease. In another scenario, where partners agree to share a minimum level of information, there would be no negative effect on the level of trust. The findings show that as the level of integration among partners increases, the need for building a trustful relationship enhances because they should share a high level of information to build trust but should also maintain confidentiality.

(2) Contracts and written agreements: all participants frequently highlighted the importance of contracts in interorganizational relationships. It seems that contracts play a major role when partners encounter challenges in their relationship. For example, as one of our respondents claimed, when a partner requires more information in order to operate the mobile plant, the decision of sharing information depends on the contract terms. One respondent stated: "that has to be negotiated like any other part of the contract". Contracts are legal mechanisms that clearly define the terms of interorganizational relationships. Even when a high level of trust exists among partners, an assurance of trust may be required by formal agreements and organizational responsibilities (Beuve and Saussier, 2012; Yuan et al., 2018). But these studies did not show that contracts can serve as a mediating variable between information sharing and trust. As the other participant claimed "if it is clearly defined in the contract that certain information is not shared, then I think it has no negative impact on the trust".

(3) Promoting transparency and information clarity: Transparency generates trust, particularly when partners have open and continuous communication and the sharing of required information takes place. Transparency implies the degree to which the MSC's targets, responsibilities, definition and level of integration are explicit and exchanged by all stakeholders and partners involved in the project (Barrane et al., 2020). To increase transparency, respondents suggested focusing on legal agreements, building a definite protocol of roles and responsibilities and developing a secure sharing of information. All these efforts help to build a trusting collaboration. Information transparency was highlighted as an important factor in creating trust and maintaining commitments by one of our respondents "If there is some ambiguity about what information is shared, then it can become a trust issue."

(4) Long-term relationship and experience: participants claimed that long-term relationships and experience with stakeholders facilitate the trust-building process, and that trust results in greater relationship investment. Partners with a high level of collaboration share risks and benefits as well as profits with trusted partners who do not act opportunistically (Ndubisi, 2010). In this regard, a participant remarked: "trust is a level of engagement between two partners that is based on several experiences".

(5) Collaboration with suitable partners: in MSCs, various stakeholders need to collaborate in order to add value to the projects. These stakeholders vary from clients, suppliers, research centers and universities and functional internal groups, to operational, tactical and strategic managers. In the context of collaboration, trust depends on each partner's experiences, competencies, attitude and role in the relationship. Therefore, partners with the proper combination of these characteristics infuse reliance and are trusted by participants in the network to add significant value in MSCs. In the same context, one of the participants noted that "we need good partners ... he has to help us otherwise we have a problem". As claimed by the participants, the current situation of MSCs, where significant research still needs to be undertaken, necessitates selecting proper research groups from universities or research institutes. 
EJMBE 31,3

\section{8}

(6) Mutual understanding of culture and language: some participants mentioned "culture" and "language" as critical elements for building trustful collaboration. This finding is consistent with the fundamentals of MSCs, where globalization is a key component. The basis of the MSCs concept is moving mobile factories to different locations close to the end customers. These mobile factories can even be transported across national borders. In this regard, the local culture and language are crucial for building trust in individuals and groups working together remotely.

The respondents stressed the effect of trust on collaboration (“. . . that is necessary to cooperate ...") and relationship investment. As the concept of MSCs is a new approach in manufacturing and modular production, there is great demand for further research and studies. The findings here show that building and maintaining mutual interorganizational trust can further increase the level of investment in both the relationship and in the research projects. This increasing investment can lead to better understanding and implementation of the concept of MSCs.

The results illustrated that trust plays a major role in establishing strategic collaboration among stakeholders in MSCs as the respondents emphasized the critical role of trust in building a long-term relationship with their partners. One of the respondents stated that “... in an MSC, you have to work much closer because you have many more interfaces with your partner and that will only work if there is a common understanding and trust in each other". Most of the respondents agreed that the level of integration with their partners would be higher when they employ mobile factories in their manufacturing process, which leads to more complicated relationships. Another respondent has mentioned that "the level of confidence and trust has to be much higher than when you are just providing products". This finding shows that the role of trust in MSCs is more significant than in traditional supply chains. The reason is that multi-stakeholders in MSCs cannot simply leave the relationship. In a traditional supply chain, when the product's quality is low, the buyer can simply terminate the relationship and select another supplier; however, in MSCs, due to the high level of integration and asset investment, the partners' propensity to leave is low. Therefore, they need to build a higher level of trust in their interorganizational relationship.

Comparing the results from the reviewed articles and semi-structured interviews, we noticed several similarities and differences in interorganizational trust between traditional supply chains and mobile supply chains. The findings show that, although three dimensions of trust in MSCs is similar to previous studies, the most significant component of trust, benevolence, was not defined in MSCs. This could be explained by the complex nature of MSCs, which requires one party to stick to the contracts, have the ability to fulfill the firm's expectation and keep their promises as one small act of opportunistic behavior can result in large risk exposure. Therefore, the benevolence component, which means the partner considers the goodwill and interest of the firm, has a rather unimportant role in trust dimensions in MSCs. In addition, this study shows that the level of trust is higher in MSCs than traditional supply chains. The reason for that is the higher level of integration between partners. Moreover, we categorized the factors of trust based on the existing literature into four groups; however, the findings show that the firms should employ different strategies for establishing trust with their partners when they are entering into the MSCs, and the previous research cannot fulfill this requirement. This approach confirms the benefit of expert interviews, i.e. the uncovering of aspects not previously considered by the researchers.

\section{Conclusion and research contributions}

This study represents a contribution to the research of the nature of trust and its elements in multi-stakeholder collaboration. The research enabled the identification of the dimensions and elements of interorganizational trust from both the existing literature and from conducted semi-structured interviews. In the first stage of analysis, 17 empirical and review 
papers revealed various differences in both the operationalization and antecedents of building interorganizational trust. The results provided in this study clustered these dimensions and antecedents of trust to build a framework in order to understand the phenomenon of trust in the interorganizational context. In the second stage, a semi-structured interview with two qualified managers from the pharmaceutical industry and one expert from academia allowed us to gain more knowledge about the nature of trust and its elements.

The study makes major theoretical contributions to the supply chain trust literature by providing a conceptual model that can serve as a basis for further empirical studies on interorganizational trust in the context of MSCs as well as multi-stakeholder collaboration. This study contributes to the present literature by showing how firms can establish trust in the context of stakeholder collaboration, such as within MSCs. It also shows the significant differences between traditional supply chains and mobile supply chains regarding the conceptualization of trust as well as the strategies of establishing trust. As suggested in previous studies, trust supports long-term interorganizational relationships and defines the duration and strength of marketing relationships (Barrane et al., 2020). This study confirms this line of argument and further emphasizes the critical role of trust in strategic collaboration in MSCs, in addition to revealing six factors or approaches for building trust in the context of MSCs: continuous communication and information sharing, contracts and written agreements, promotion transparency and information clarity, long-term relationship and experience, selecting suitable partners and mutual understandings of culture and language. The findings highlight the importance of two major outcomes of long-lasting trust in multistakeholder MSCs, namely collaboration and relationship investment. The study adds to the body of knowledge in building trust in multiple stakeholder collaboration, particularly in innovative organizations which are involved with disruptive technologies.

As for the practical and managerial field, this study contributes in several ways. This study evaluates the trusting collaboration of multiple stakeholders. Since the concept of MSCs is somewhat novel, the body of the available literature is still somewhat limited. This study contributes to the advancement of research in this area by examining various information-sharing scenarios of MSCs and identifying the role of trust in this concept. Interorganizational collaborations in MSCs are more successful when saturated with trust. The collaboration systems must allow the innovative organizations to create value in a trusting environment through the adaptation of advanced technologies without failure. Overall, this study contributes to organizational communication and will thus be beneficial to communication scientists and supply chain managers considering trust-building measures as the integration of multiple stakeholders was considered and examined.

However, the results of our study should be interpreted keeping in mind several limitations that may guide future research. First, considering a limited number of participants in the semi-structured interview limits the generalizability of the results. Thus, we suggest that future research should employ a greater number of respondents. Second, considering one industry for our study may require caution when generalizing the findings. Furthermore, applying qualitative methodologies for analysis limits the generalizability of the results. Future studies are recommended to employ mixed-method approaches in order to analyze the findings both qualitatively and quantitatively. We suggest that future studies employ the conceptual model presented in this study in other industries to obtain more understanding of its validity and generalizability of the findings.

\section{References}

Agarwal, U.A. and Narayana, S.A. (2020), "Impact of relational communication on buyer-supplier relationship satisfaction: role of trust and commitment", Benchmarking: An International Journal, Vol. 27 No. 8, pp. 2459-2496.
Factors and outcomes of trust in MSCs 
EJMBE 31,3

\section{0}

Akrout, H. and Diallo, M.F. (2017), "Fundamental transformations of trust and its drivers: a multistage approach of business-to-business relationships", Industrial Marketing Management, Vol. 66, pp. 159-171.

Alix, T., Benama, Y. and Perry, N. (2019), "A framework for the design of a reconfigurable and mobile manufacturing system”, Procedia Manufacturing, Vol. 35, pp. 304-309.

Allman, A. and Zhang, Q. (2020), "Dynamic location of modular manufacturing facilities with relocation of individual modules", European Journal of Operational Research, Vol. 286 No. 2, pp. 494-507.

Arvidsson, A. and Melander, L. (2020), "The multiple levels of trust when selecting suppliers - insights from an automobile manufacturer", Industrial Marketing Management, Vol. 87, pp. 138-149.

Aslam, J., Saleem, A., Khan, N.T. and Kim, Y.B. (2021), "Factors influencing blockchain adoption in supply chain management practices: a study based on the oil industry", Journal of Innovation and Knowledge, Vol. 6 No. 2, pp. 124-134.

Baldea, M., Edgar, T.F., Stanley, B.L. and Kiss, A.A. (2017), "Modular manufacturing processes: status, challenges, and opportunities", AIChE Journal, Vol. 63 No. 10, pp. 4262-4272.

Barrane, F.Z., Ndubisi, N.O., Kamble, S., Karuranga, G.E. and Poulin, D. (2020), "Building trust in multi-stakeholder collaborations for new product development in the digital transformation era”, Benchmarking: An International Journal, Vol. 28 No. 1, pp. 205-228.

Becker, T., Lier, S. and Werners, B. (2019), "Value of modular production concepts in future chemical industry production networks", European Journal of Operational Research, Vol. 276 No. 3, pp. 957-970.

Beuve, J. and Saussier, S. (2012), "Interfirm cooperation in strategic relationships: the role of formal contract", Industrial and Corporate Change, Vol. 21 No. 4, pp. 811-836.

Cagliano, A.C., De Marco, A. and Rafele, C. (2017), "E-grocery supply chain management enabled by mobile tools", Business Process Management Journal, Vol. 23 No. 1, pp. 47-70.

Cappiello, G., Giordani, F. and Visentin, M. (2020), "Social capital and its effect on networked firm innovation and competitiveness", Industrial Marketing Management, Vol. 89, pp. 422-430.

Chan, F.T. and Yee-Loong Chong, A. (2013), "Determinants of mobile supply chain management system diffusion: a structural equation analysis of manufacturing firms", International Journal of Production Research, Vol. 51 No. 4, pp. 1196-1213.

Chen, J.V., Yen, D.C., Rajkumar, T.M. and Tomochko, N.A. (2011), "The antecedent factors on trust and commitment in supply chain relationships", Computer Standards and Interfaces, Vol. 33 No. 3, pp. 262-270.

Daudi, M., Hauge, J.B. and Thoben, K.-D. (2016), "Behavioral factors influencing partner trust in logistics collaboration: a review", Logistics Research, Vol. 9 No. 1, pp. 1-11.

Delbufalo, E. (2012), "Outcomes of inter-organizational trust in supply chain relationships: a systematic literature review and a meta-analysis of the empirical evidence", Supply Chain Management: An International Journal, Vol. 17 No. 4, pp. 377-402.

Driessen, P.H. and Hillebrand, B. (2013), "Integrating multiple stakeholder issues in new product development: an exploration", Journal of Product Innovation Management, Vol. 30 No. 2, pp. 364-379.

Eng, T.Y. (2006), "An investigation into the mediating role of cross-functional coordination on the linkage between organizational norms and SCM performance", Industrial Marketing Management, Vol. 35 No. 6, pp. 762-773.

Fawcett, S.E., Jin, Y.H., Fawcett, A.M. and Magnan, G. (2017), "I know it when I see it: the nature of trust, trustworthiness signals, and strategic trust construction”, The International Journal of Logistics Management, Vol. 28 No. 4, pp. 914-938.

Fernando, Y. and Wulansari, P. (2020), "Perceived understanding of supply chain integration, communication and teamwork competency in the global manufacturing companies", European Journal of Management and Business Economics, Vol. 30 No. 2, pp. 191-210. 
Ford, J.K., Riley, S.J., Lauricella, T.K. and van Fossen, J.A. (2020), "Factors affecting trust among natural resources stakeholders, partners, and strategic alliance members: a meta-analytic investigation", Frontiers in Communication, Vol. 5 No. 9.

Fox, S. (2015), "Moveable factories: how to enable sustainable widespread manufacturing by local people in regions without manufacturing skills and infrastructure", Technology in Society, Vol. 42, pp. 49-60.

Fox, S. (2019), "Moveable production systems for sustainable development and trade: limitations, opportunities and barriers", Sustainability, Vol. 11 No. 19, p. 5154.

Frydlinger, D., Hart, O. and Vitasek, K. (2019), “A new approach to contracts”, Harvard Business Review, Vol. 97 No. 5, pp. 116-126.

Haghjoo, N., Tavakkoli-Moghaddam, R., Shahmoradi-Moghadam, H. and Rahimi, Y. (2020), "Reliable blood supply chain network design with facility disruption: a real-world application", Engineering Applications of Artificial Intelligence, Vol. 90, p. 103493.

Handfield, R.B. and Bechtel, C. (2002), "The role of trust and relationship structure in improving supply chain responsiveness", Industrial Marketing Management, Vol. 31 No. 4, pp. 367-382.

Hedelind, M., Jackson, M., Funk, P., Stahre, J., Söderberg, R., Carlsson, J., Björkman, M. and Winroth, M. (2007), "Factory-in-a-Box - solutions for availability and mobility of flexible production capacity", Proceedings of Swedish Production Symposium, Vol. 7, pp. 28-30.

Jackson, M., Wiktorsson, M. and Bellgran, M. (2008), "Manufacturing systems and technologies for the new frontier", Manufacturing Systems and Technologies for the New Frontier, Springer, London, pp. 341-346.

Kuckartz, U. and Rädiker, S. (2019), "Working with coded segments and memos", Analyzing Qualitative Data with MAXQDA, Springer, Cham, doi: 10.1007/978-3-030-15671-8_9.

Kwon, I.-W.G. and Suh, T. (2004), "Factors affecting the level of trust and commitment in supply chain relationships", The Journal of Supply Chain Management, Vol. 40 No. 2, pp. 4-14.

Lee, L.S. and Zhong, W. (2020), "Dependence structure, trust dimensions, and governance choices in Asian marketing channels: evidence in China”, Asian Journal of Business Research, Vol. 10 No. 1, p. 48.

Lohtander, M., Aholainen, A., Volotinen, J., Peltokoski, M. and Ratava, J. (2017), "Location independent manufacturing - case-based blue ocean strategy", Procedia Manufacturing, Vol. 11, pp. 2034-2041.

Lui, S.S. and Ngo, H.-Y. (2004), "The role of trust and contractual safeguards on cooperation in nonequity alliances", Journal of Management, Vol. 30 No. 4, pp. 471-485.

Matt, D.T., Rauch, E. and Dallasega, P. (2015), "Trends towards distributed manufacturing systems and modern forms for their design", Procedia CIRP, Vol. 33, pp. 185-190.

Mirkouei, A., Mirzaie, P., Haapala, K.R., Sessions, J. and Murthy, G.S. (2016), "Reducing the cost and environmental impact of integrated fixed and mobile bio-oil refinery supply chains", Journal of Cleaner Production, Vol. 113, pp. 495-507.

Morgan, R.M. and Hunt, S.D. (1994), “The commitment-trust theory of relationship marketing”, Journal of Marketing, Vol. 58 No. 3, pp. 20-38.

Ndubisi, N.O. (2010), "Conflict handling, trust and commitment in outsourcing relationship: a Chinese and Indian study", Industrial Marketing Management, Vol. 40 No. 1, pp. 109-117.

Ndubisi, N.O., Dayan, M., Yeniaras, V. and Al-hawari, M. (2020), "The effects of complementarity of knowledge and capabilities on joint innovation capabilities and service innovation: the role of competitive intensity and demand uncertainty", Industrial Marketing Management, Vol. 89, pp. 196-208.

Nematollahi, I. (2019), "Empirical investigation of trust antecedents and consequences in decentralized supply chain: the case of cosmetics market in Iran", Decision Science Letters, Vol. 8 No. 4, pp. 483-504.
Factors and outcomes of trust in MSCs 
EJMBE 31,3

\section{2}

Nicolaou, A.I. and McKnight, D.H. (2006), "Perceived information quality in data exchanges: effects on risk, trust, and intention to use”, Information Systems Research, Vol. 17 No. 4, pp. 332-351.

Nyaga, G.N., Whipple, J.M. and Lynch, D.F. (2010), "Examining supply chain relationships: do buyer and supplier perspectives on collaborative relationships differ?", Journal of Operations Management, Vol. 28 No. 2, pp. 101-114.

Öberg, C. (2019), "Additive manufacturing - digitally changing the global business landscape", European Journal of Management and Business Economics, Vol. 28 No. 2, pp. 174-188.

Oghazi, P., Fakhrai Rad, F., Karlsson, S. and Haftor, D. (2018), "RFID and ERP systems in supply chain management", European Journal of Management and Business Economics, Vol. 27 No. 2, pp. 171-182.

Olugboyega, O. and Windapo, A. (2019), "Theoretical model of trust-based relationships in building information modelling supply chain for construction projects", Acta Structilia, Vol. 26 No. 2, pp. 107-141.

Palaniappan, G. (2017), "Determinants of corporate financial performance relating to board characteristics of corporate governance in Indian manufacturing industry: an empirical study", European Journal of Management and Business Economics, Vol. 26 No. 1, pp. 67-85.

Paluri, R.A. and Mishal, A. (2020), "Trust and commitment in supply chain management: a systematic review of literature", Benchmarking: An International Journal, Vol. 27 No. 10, pp. 2831-2862.

Pan, Y., Nam, T., Ogara, S. and Lee, S. (2013), "Adoption model of mobile-enabled systems in supply chain mobile-enabled systems in supply chain”, Industrial Management and Data Systems, Vol. 113 No. 2, pp. 263-5577.

Pech, W. and Swicegood, P. (2013), "Trust and trustworthiness: a game theory transcontinental experiment", International Business and Economics Research Journal (IBER), Vol. 12 No. 3, p. 311.

Peltokoski, M., Volotinen, J. and Lohtander, M. (2017), "Location independent manufacturing - a software solution for supply chains”, Procedia Manufacturing, Vol. 11, pp. 863-870.

Pomponi, F., Fratocchi, L. and Tafuri, S.R. (2015), "Trust development and horizontal collaboration in logistics: a theory based evolutionary framework", Supply Chain Management, Vol. 20 No. 1, pp. 83-97.

Rosell, J. (2004), "Assembly and task planning using Petri nets: a survey", Proceedings of the Institution of Mechanical Engineers, Part B: Journal of Engineering Manufacture Vol. 218 No. 8, pp. 987-994.

Ryciuk, U. (2017), "Identification of factors related to trust formation in construction supply chains", Procedia Engineering, Vol. 182, pp. 627-634.

Salam, M.A. (2017), "The mediating role of supply chain collaboration on the relationship between technology, trust and operational performance: an empirical investigation", Benchmarking, Vol. 24 No. 2, pp. 298-317.

Sarkar, S., Chauhan, S. and Khare, A. (2020), "A meta-analysis of antecedents and consequences of trust in mobile commerce", International Journal of Information Management, Vol. 50, pp. 286-301.

Savastano, M., Amendola, C. and D'Ascenzo, F. (2018), "How digital transformation is reshaping the manufacturing industry value chain: the new digital manufacturing ecosystem applied to a case study from the food industry", Network, Smart and Open, Springer, Cham, pp. 127-142.

Shahmoradi-Moghadam, H. and Schönberger, J. (2021), "Joint optimization of production and routing master planning in mobile supply chains", Operations Research Perspectives, Vol. 8, p. 100187.

F3 factory (2013), "Fast, flexible, modular production technology provides platform for future European growth", available at: https://cordis.europa.eu/docs/results/228867/final1-f3-factorykeymessages-and-casestudy-summaries.pdf (accessed 2 May 2021). 
Wooliscroft, B. and Ganglmair-Wooliscroft, A. (2018), "Growth, excess and opportunities", Journal of Macromarketing, Vol. 38 No. 4, pp. 355-363.

Wu, G., Zhao, X. and Zuo, J. (2017), "Relationship between project's added value and the trust-conflict interaction among project teams", Journal of Management in Engineering, Vol. 33 No. 4, p. 4017011.

Yang, J., Wang, J., Wong, C.W.Y. and Lai, K.-H. (2008), "Relational stability and alliance performance in supply chain”, Omega, Vol. 36 No. 4, pp. 600-608.

Yuan, Y., Feng, B., Lai, F. and Collins, B.J. (2018), "The role of trust, commitment, and learning orientation on logistic service effectiveness", Journal of Business Research, Vol. 93, pp. 37-50.

Zafari, K., Biggemann, S. and Garry, T. (2020), "Mindful management of relationships during periods of crises: a model of trust, doubt and relational adjustments", Industrial Marketing Management, Vol. 88, pp. 278-286.

Zhong, W., Su, C., Peng, J. and Yang, Z. (2017), “Trust in interorganizational relationships”, Journal of Management, Vol. 43 No. 4, pp. 1050-1075.

\section{Appendix 1}

\section{Semi-structured interview questions:}

(1) Could you please explain more about MSCs projects in your company?

(2) We would like to know how you define trust in the concept of MSCs.

(3) What can help you to maintain a trustworthy relationship with your stakeholders?

(4) Do you think there would be any differences in trust between partners with this concept?

(5) Do you think refusing to share a piece of information could affect your partner's trust level? Could it affect your informal relationship?

\section{Appendix 2}

\section{The procedure of identifying reviewed articles}

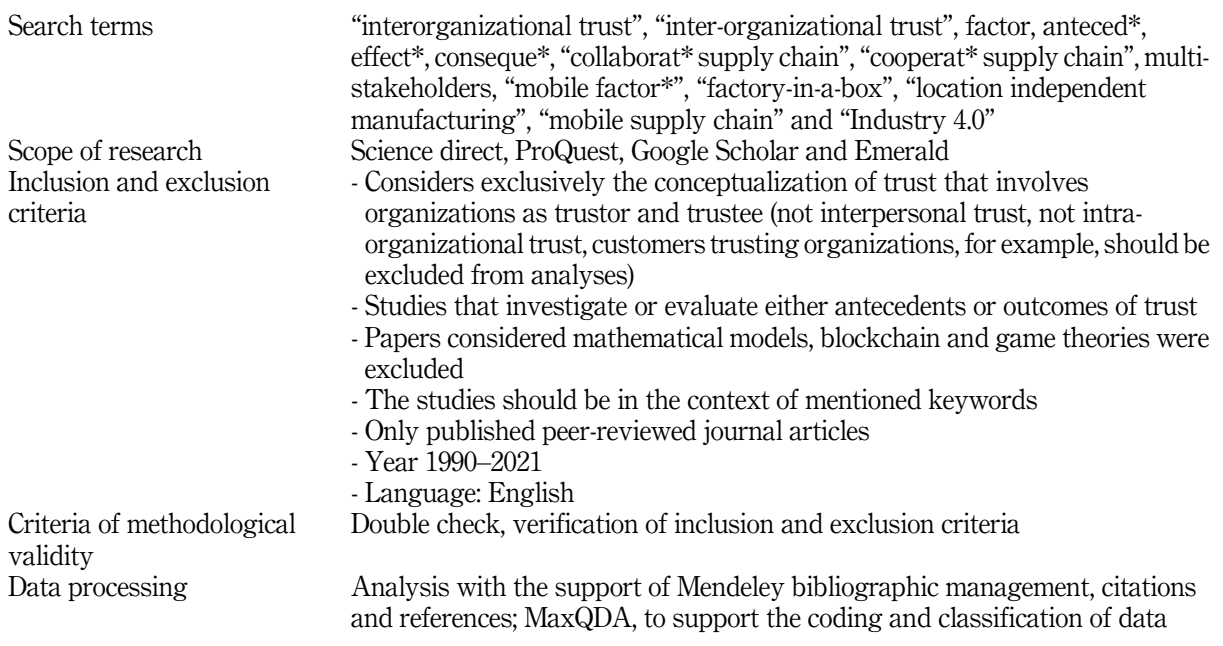

Factors and outcomes of trust in MSCs 
EJMBE

31,3

\section{4}

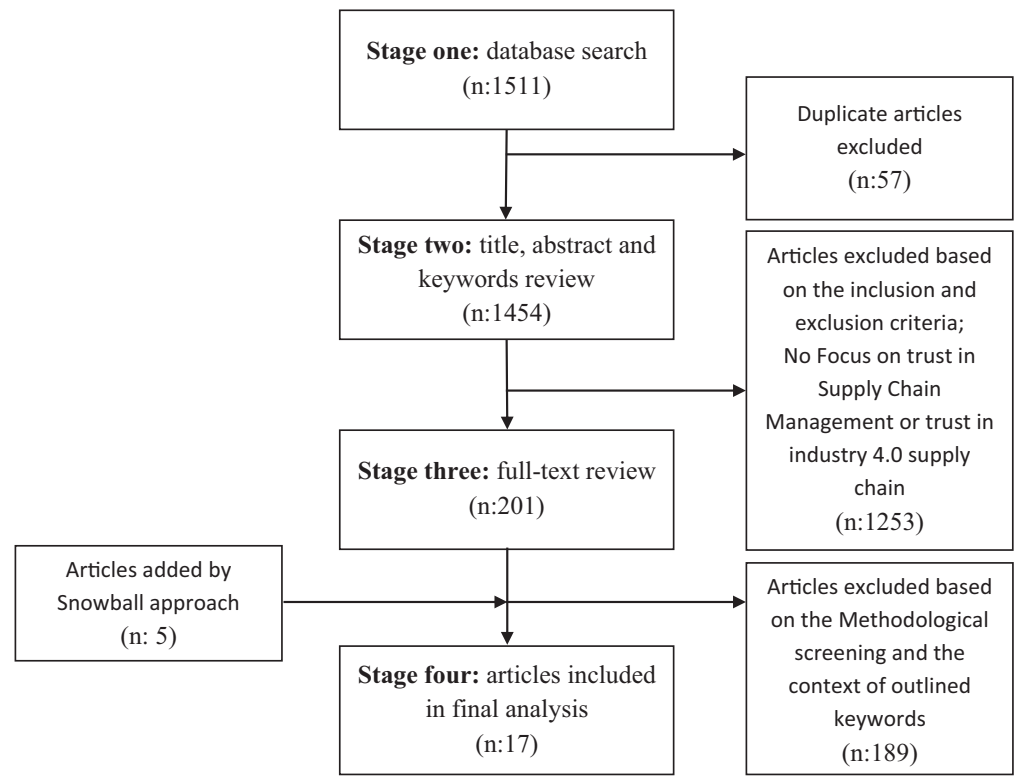

Corresponding author

Nassim Ghondaghsaz can be contacted at: nassim.ghondaghsaz@tu-dresden.de

For instructions on how to order reprints of this article, please visit our website:

www.emeraldgrouppublishing.com/licensing/reprints.htm

Or contact us for further details: permissions@emeraldinsight.com 